\title{
Growing Human Embryonic Neurons in Glass Capillary: A Simple Method to Study Interaction between Motile Cells and Neurons and to Explore the Neurotoxicity of Metals
}

\author{
M. Stabile \\ Zigote Center, Salerno, Italy \\ Email: zigote@tiscali.it
}

Received 29 August 2014; revised 26 September 2014; accepted 12 October 2014

Copyright (C) 2014 by author and Scientific Research Publishing Inc.

This work is licensed under the Creative Commons Attribution International License (CC BY).

http://creativecommons.org/licenses/by/4.0/

(c) $\underset{\mathrm{EY}}{\mathrm{C}}$ Open Access

\begin{abstract}
A new method of growing human embryonic neurons in glass capillary is proposed. Beside the simplicity of the method, the main advantages are represented by the possibility to study the interactions between a directional flow of human motile cells (leukocytes, red blood cells, sperm cells) and the neuronal monolayer and to evaluate the toxicity on neuronal growth of a copper wire or others filiform materials introduced in the glass capillary. The neurotoxicity of copper is verified by this device, because copper wire inserted into the capillary interferes with engraftment and growth of neuronal cells. No interference by neuronal monolayer was found with the flow of human sperms trough the capillary. Also the flow of leukocytes and erythrocytes occurred without agglutination or adhesion to cellular monolayer with serial observation at 30, 60, 120 minutes. A further advantage of this method is the possibility of withdrawing culture medium microquantities from capillary in order to study neuronal secretory activity.
\end{abstract}

\section{Keywords}

Human Embryonic Neurons, Neuronal Growth in Glass Capillary, Copper Neurotoxicity

\section{Introduction}

The neuronal monolayer culture can be performed in the traditional plastic flasks, alternatively other culture kits 
have been used in order to evaluate neuronal migration, apoptosis and response to neurotrophic, chemo-tactical factors:

- Cell Migration Assay: the 96 well assay platform uses stoppers to create a central exclusion zone; cells are plated outside the zone and migrate once the stopper is removed. The presence of neurotoxicant, neurotrophic or neurokinetic factors in the wells can be comparatively studied, analyzing the migration of neural stem cells in the central zone of the well [1].

- Neurospheres as Three-Dimensional Cellular Systems [2] (Figure 1).

Thermo Scientific Chamber Slides: the chamber slides simplify imaging analysis and cytostaining process. The multi-chamber kit allows for parallel studies of multiple conditions and stimuli required by many applications [3].

A new method of neuronal culture in glass capillary is proposed, whose advantages are represented by:

- Simplicity;

- possibility of promoting neuronal growth which physiologically occurs along columns rather than in radial direction;

- small irregularities of the glass surface such as grooves and ridges can affect the direction of axonal growth when the structures are of micron sizes [4]; the presence of small bumps in a capillary tube can further promote the growth in the direction of the length of the tube, making it more evident factors stimulating or interfering with growth and migration;

- introducing a copper wire or other metal filiform materials in the glass capillary, it is possible to evaluate the toxicity on neuronal growth;

- the possibility to assess the interaction between a directional flow of human motile cells (leukocytes, red blood cells, sperm cells) through the glass capillary and the neuronal monolayer;

- the possibility to collect microquantity of liquid from the capillary for biochemical assay.

\section{Material and Methods}

\subsection{Cell Culture in Glass Capillary}

A culture of embryonic neurons derived from human embryos of 7 - 10 weeks was prepared according to the procedures used in our laboratory [5]. A confluent neuronal monolayer was treated with mild trypsinization (1 cc of $0.05 \%$ trypsin-EDTA Gibco for $1 \mathrm{~min}$ ); after washing, neurons detached from the monolayer were suspended in 0.5 cc of Chang Medium (Irvine Scientific), were aspirated into a 1 cc syringe and injected into a clean, sterilized glass microhematocrit capillary tube. The capillary was placed in a plastic Petri dishes (Falcon $35 \times 10 \mathrm{~mm}$ ) with $3 \mathrm{ml}$ Chang medium (Figure 2(A) and Figure 2(B)).

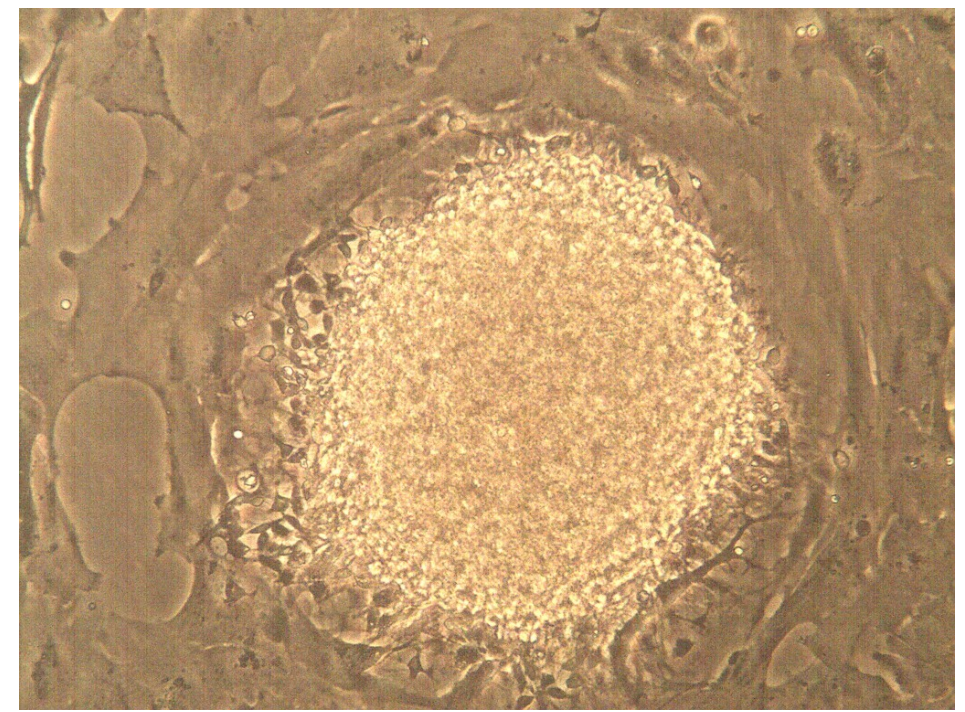

Figure 1. Neurosphera: a globular neuronal mass visible in human embryonic neuronal culture (personal observation Dr. Stabile). 

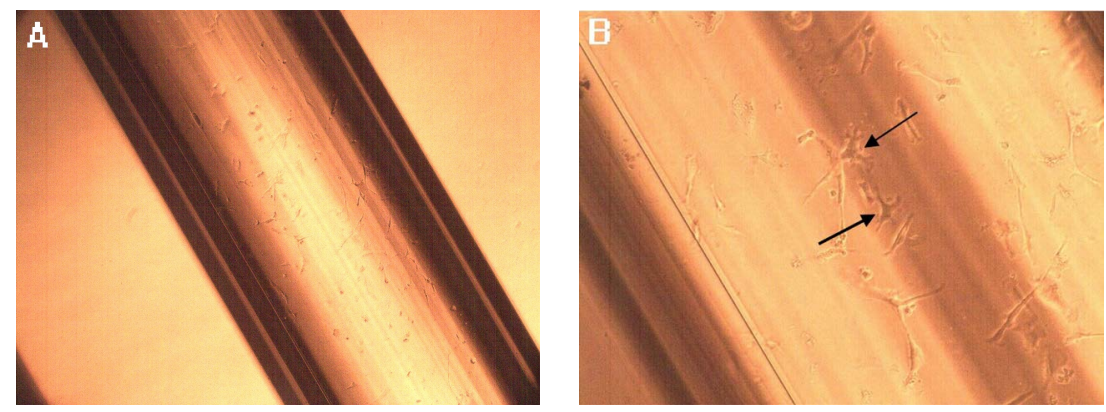

Figure 2. Neuronal growth in glass capillary tube: observation at phase contrast microscopy with magnification of $200 \times$ (A) and $400 \times$ (B) Arrows point to neurons.

\subsection{Culture Preparation in Capillaries with a Copper Wire Inserted (Figure 3)}

The modification compared to the previously described procedure consisted of inserting a copper wire inside the glass capillary.

\subsection{Evaluation of Flow of Motile Cells (Sperm, White Blood Cells, Red Blood Cells) through Glass Capillary Coated with a Neuronal Monolayer of Neurons}

Human semen of normospermic subjects washed and diluted 1:5 with QUINN'S ${ }^{\circledR}$ Sperm Washing Medium was placed in the plastic dish with the capillary coated with a neuronal monolayer.

Blood samples $(3 \mathrm{ml})$ of three different individuals without hematological diseases were treated with Histopaque-1077 (Sigma) to isolate the leukocytes. The final leukocyte pellet was suspended in RPM1 and 0.5 cc of the final suspension was injected with insulin syringe in the glass capillary coated with neuronal monolayer. In addition 0.5 cc of heparinized whole blood from the same subject, diluted 1:20 with physiological solution, was similarly injected with insulin syringe in the glass capillary coated by neuronal monolayer.

\subsection{NSE Biochemical Assay in Medium Culture}

The neurono specific enolase (NSE), the glycolytic enolase isoenzyme, is a specific product of neurons and neuroendocrine cells. Its presence in the culture medium is therefore a confirmation of the neuronal cell type and can be expression of the secretory activity. The NSE was assayed by ELISA (Enolasi Neurone Specifica ELISA Kit, Inter Medical S.r.l. Italy) in both the medium surrounding the capillary and in the liquid inside the capillary aspirated with microsyringe. A concentration of NSE of $0.21 \mu \mathrm{g} / \mathrm{l}$ was present in the medium recovered from the capillary.

\section{Results}

After a period of 7 days, neuronal growth was observed with phase contrast microscope on the capillary inner surface; within 2 weeks, the neuronal coating was almost complete (Figure 4).

\subsection{Test with Copper Wire inside the Capillary}

The absence of engraftment and cell growth, likely due to the neuro-toxicity of copper, was recorded inside glass capillaries inserted with copper wire in 3 repeated attempts to set up a neuronal colture (Figure 5).

\subsection{Test with Human Semen Injected into the Capillary}

Phase contrast observation showed the passage of motile sperm through the capillary without adhesion of sperm to the monolayer or reduction in sperm motility. The non-interference between the two cell types was confirmed repeating 3 times the experiment (Figure 6(a) and Figure 6(b)).

\subsection{Test with Whole Human Blood or Isolated Leukocytes into the Capillary}

The microscopic observation showed the passage of leukocytes and erythrocytes without agglutination or adhe- 


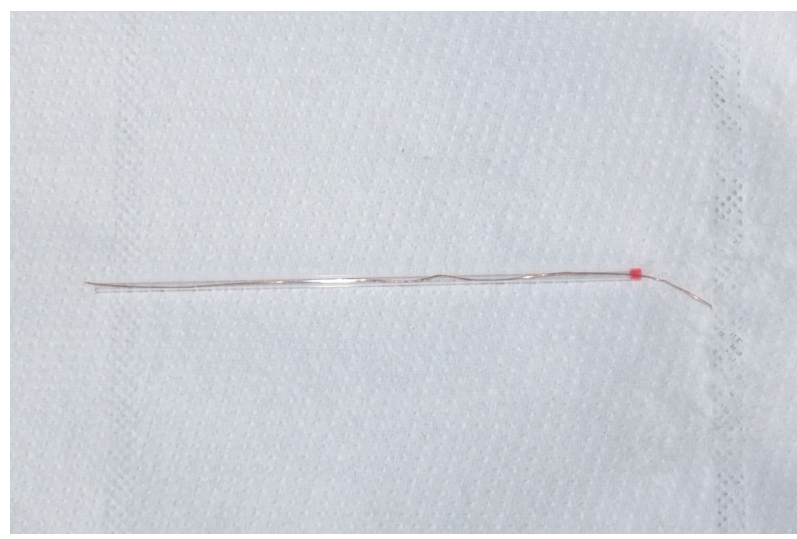

Figure 3. The capillary tube with a wire copper inserted.

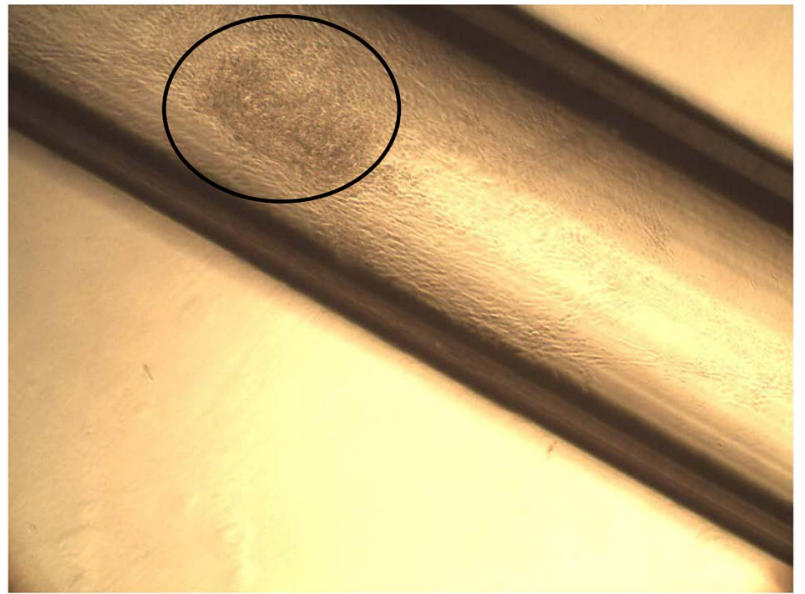

Figure 4. Neuronal growth observation after 7 days: an almost complete coating neuronal layer is observable on the inner surface of the capillary. The circle delimits a growth confluent zone.

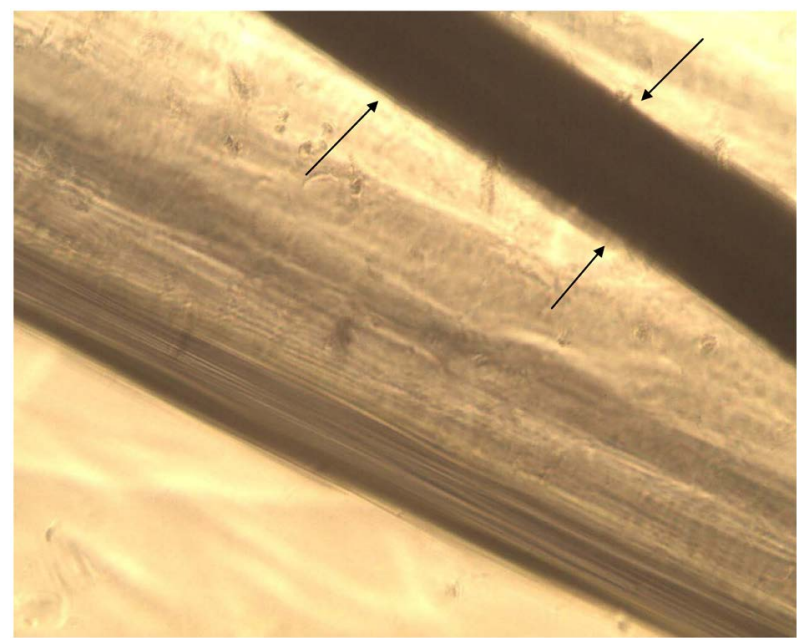

Figure 5. Capillary with the copper wire inside: the copper wire is observable on the upper of the microscopic image (arrows); only destroyed cells and no neuronal growth are observed. 
sion to the neuronal monolayer with serial observation at 30, 60, 120 minutes. After 24 hours the red blood cells appeared sedimented on the bottom of the capillary without adherence to neuronal monolayer (Figure 7(a) and Figure 7(b)).

\section{Discussion}

A toxic role of copper on neuronal growth may be inferred from experiments conducted with neuronal growth in glass capillary. There are numerous studies that correlate an increase in blood level of free copper not bound to ceruloplasmin with Alzheimer's Disease (AD) [6]-[8]. Copper takes part in a variety of biological reductionoxidation (redox) processes; ceruloplasmin, the copper-transporting protein, also possesses an important redox capacity. AD patients show abnormally high concentration of $\mathrm{Cu}(2+)$ in the amyloid $\beta(\mathrm{AB})$ plaques; the $\mathrm{AB}$ : copper complexes can catalytically oxidize cholesterol to generate $\mathrm{H}_{2} \mathrm{O}_{2}$, oxysterols and other lipid peroxidation products that accumulate in brains of AD patients [9]-[11]. In analogy with what was assumed in Alzheimer's disease, the neurotoxic role of copper on neuronal growth may lie in its ability to create superoxide. Copper cytotoxicity is already known, because of its use in intrauterine devices for contraception; mechanism of action would reside in production of cytotoxic peptides, inhibition of sperm motility and capacitation [12].

However, further studies and experiments are necessary in order to confirm the copper neurotoxicity.

The sperm passage through capillary coated by neuronal monolayer without interference with the sperm motility confirms what could be theoretically supposed, since the two cell types are not in contact in the living organism. However, a percentage of glial cells is present in the neuronal monolayer and mesenchimal cells could in theory react to foreign antigens (neurons and gametes derive from different subjects); however the exposure time is too short to allow any type of immune response.

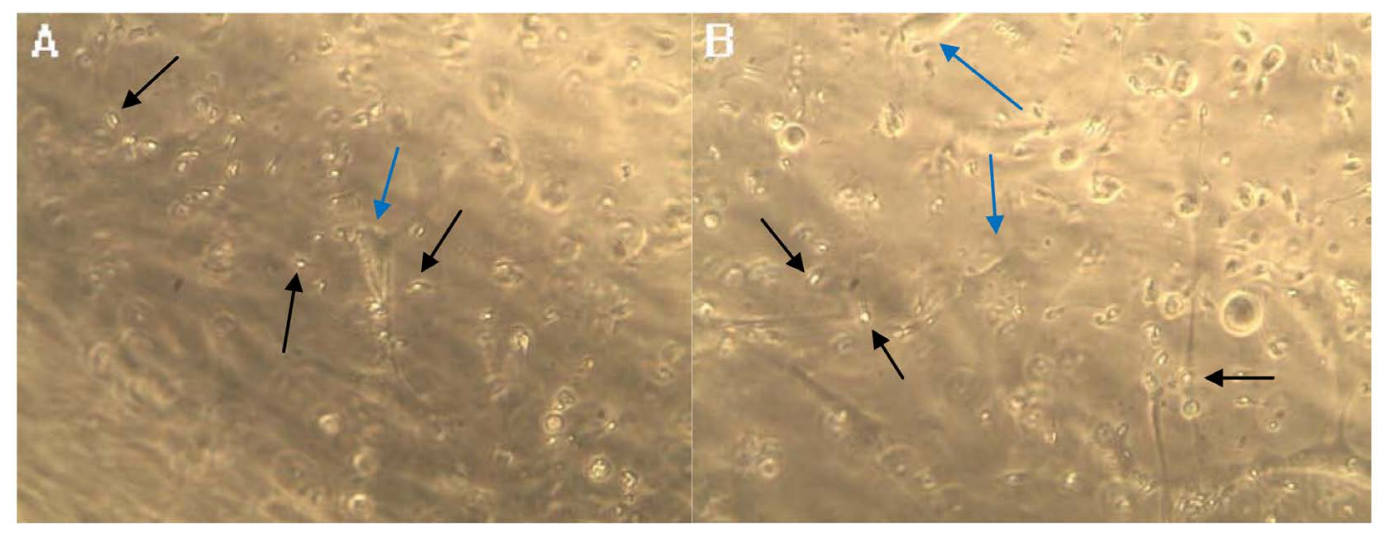

Figure 6. (A) (B) Motile sperm in the capillary: no effect on sperm motility or agglutination by the cellular monolayer is observed. Black arrows point to the heads of spermatozoa; blue arrows to neurons.

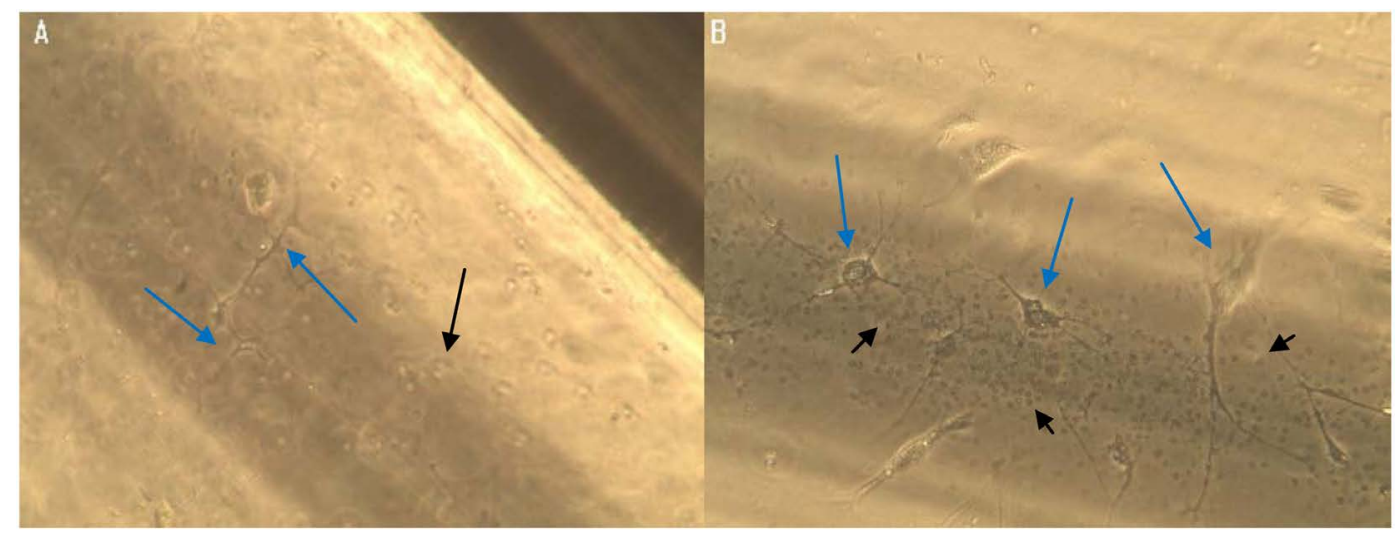

Figure 7. (A) (B) Diluted whole blood in capillary coated by neuronal monolayer at 60 minutes (A) and after $24 \mathrm{~h}$ (B): sedimented erythrocytes interspersed with neurons are observable in (B) Black arrows points to white cells and erythrocytes; blue arrows to neurons. 
Also the blood cells do not show interaction with neuronal monolayer although antigenically different; also in this case the limited observation time does not allow to exclude a more late immune and cytotoxic reaction.

\section{Conclusion}

In conclusion, the capillary tube coated by human neuronal monolayer has proven to be a simple and versatile technical device for the study of neuronal growth and its interfering factors, for the assay of substances produced by neuronal mini-factory and finally for the assessment of the interaction between neurons and other cell types in suspension.

\section{Acknowledgements}

The author thanks Dr. Lucia Marino for assistance.

\section{References}

[1] Powe Jr., A.C., et al. (2010) Identification of Stimulators and Inhibitors of Cell Migration in Human Embryonic Stem Cell Derived Neural Progenitors Using a Novel, High Throughput Amenable Assay Platform. Posters Platypus Technologies.

[2] Moors, M., et al. (2009) Human Neurospheres as Three-Dimensional Cellular Systems for Developmental Neurotoxicity Testing. Environmental Health Perspectives, 117, 1131-1138. http://dx.doi.org/10.1289/ehp.0800207

[3] Neeley, C. (2012) Optimizing Neuron Adhesion and Growth by Choosing the Right Surface of Thermo Scientific Chamber Slide. Thermo Fisher Scientific, Rochester.

[4] Baranes, K., Chejanovsky, N., Alon, N., Sharoni, A. and Shefi, O. (2012) Topographic Cues of Nano-Scale Height Direct Neuronal Growth Pattern. Biotechnology and Bioengineering, 109, 1791-1797. http://dx.doi.org/10.1002/bit.24444

[5] Stabile, M., et al. (2012) Human Embryo Neuronal Culture in Vitro: A Model to Study Cellular Physiology, Receptors, Power and Toxicity of Cytostatic Drugs for Human Use. Neuroscience \& Medicine, 3, 321-326. http://dx.doi.org/10.4236/nm.2012.33037

[6] Squitti, R., et al. (2014) Meta-Analysis of Serum Non-Ceruloplasmin Copper in Alzheimer's Disease. Journal of Alzheimer's Disease, 38, 809-822.

[7] Park, J.H., Lee, D.W. and Park, K.S. (2013) Elevated Serum Copper and Ceruloplasmin Levels in Alzheimer’s Disease. Asia-Pacific Psychiatry, 6, 38-45. http://dx.doi.org/10.1111/appy.12077

[8] Liu, H.P., et al. (2013) Genetic Variability in Copper-Transporting P-Type Adenosine Triphosphatase (ATP7B) Is Associated with Alzheimer's Disease in a Chinese Population. Journal of Biological Regulators \& Homeostatic Agents, 27, 319-327.

[9] Collin, F., Sasaki, I., Eury, H., Faller, P. and Hureau, C. (2013) Pt(II) Compounds Interplay with Cu(II) and Zn(II) Coordination to the Amyloid- $\beta$ Peptide Has Metal Specific Consequences on Deleterious Processes Associated to Alzheimer's Disease. Chemical Communications, 49, 2130-2132. http://dx.doi.org/10.1039/c3cc38537j

[10] Hung, Y.H., Bush, A.I. and La Fontaine, S. (2013) Links between Copper and Cholesterol in Alzheimer’s Disease. Frontiers in Physiology, 4. http://dx.doi.org/10.3389/fphys.2013.00111

[11] Ghosh, C. and Dey, S.G. (2013) Ligand-Field and Ligand-Binding Analysis of the Active Site of Copper-Bound A $\beta$ Associated with Alzheimer’s Disease. Inorganic Chemistry, 52, 1318-1327. http://dx.doi.org/10.1021/ic301865n

[12] Dean, G. and Schwarz, E.B. (2011) Intrauterine Contraceptives (IUCs). In: Hatcher, R.A., Trussell, J., Nelson, A.L., Cates Jr., W., Kowal, D., Policar, M.S., Ed., Contraceptive Technology (20th Revised Ed.), Ardent Media, New York, 147-191. 
Scientific Research Publishing (SCIRP) is one of the largest Open Access journal publishers. It is currently publishing more than 200 open access, online, peer-reviewed journals covering a wide range of academic disciplines. SCIRP serves the worldwide academic communities and contributes to the progress and application of science with its publication.

Other selected journals from SCIRP are listed as below. Submit your manuscript to us via either submit@scirp.org or Online Submission Portal.
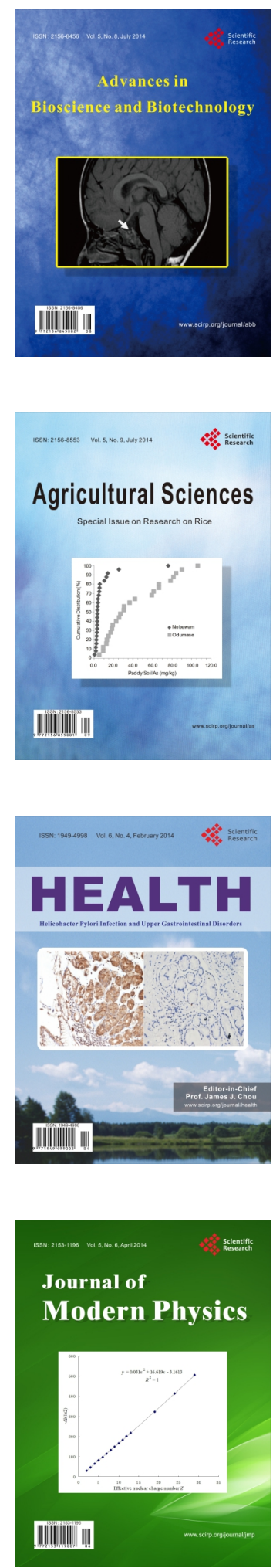
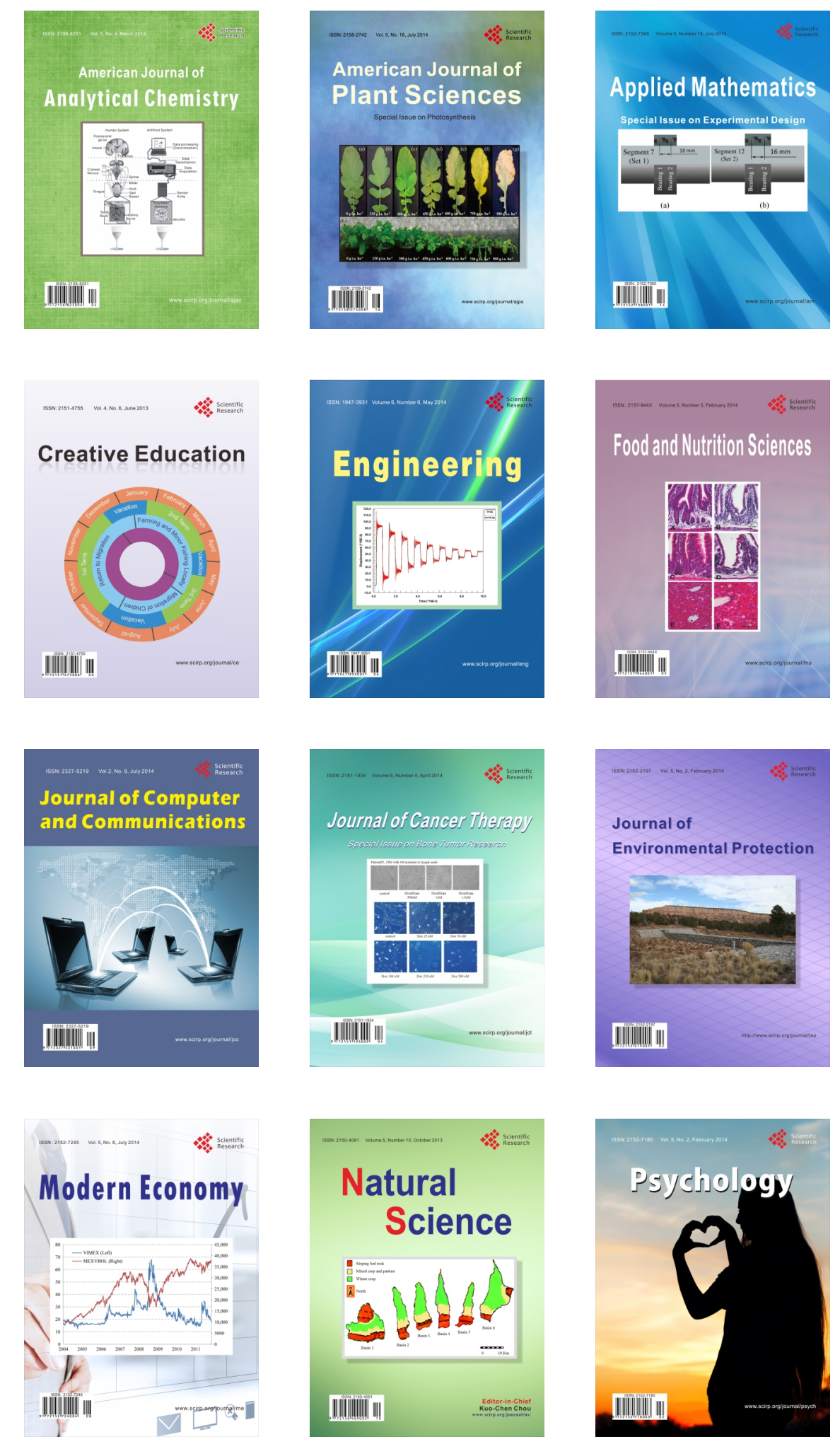